\title{
National Vulnerability Comprehensive Evaluation Model - Taking South Sudan as an Example
}

\author{
Wei Shao ${ }^{1, ~ a, ~ Z h a n g q i ~ Z h a n g, ~ b, ~ W e n t a o ~ Y a n g ~}{ }^{2, ~ c}$, Chunhui Bao ${ }^{1, d}, \mathrm{Yi} \mathrm{He}^{3, \mathrm{e}}$ \\ ${ }^{1}$ Zhejiang Yuexiu University of Foreign Languages College of International Business, Zhejiang, \\ China \\ 2 Tongji University College of Civil Engineering, China \\ ${ }^{3}$ Zhejiang Yuexiu University of Foreign Languages College of International Business College of \\ Network Communication, Zhejiang, China

$$
\begin{gathered}
\text { a 1277149583@qq.com } \\
\text { b309421912@qq.com } \\
\text { c wt1540773103@163.com } \\
\text { d354696890@qq.com } \\
\text { e1138234695@qq.com }
\end{gathered}
$$

Keywords: National vulnerability; evaluation model; quantification.

\begin{abstract}
National Vulnerability Assessment is a comprehensive concept used to describe the development and decline of a country. It aims at certain human activities and makes detailed judgments and predictions on the degree of pollution of an environmental system through scientific methods. This paper takes the South Sudan country as a case study and uses the correlation coefficient model to analyze the impact of the rising average temperature on the vulnerability of the country. Through ranking and comparative analysis of cumulative values of national vulnerability assessment indicators. Taking the impact of global warming into account, a comprehensive national vulnerability assessment model was used to quantify this change.
\end{abstract}

\section{Introduction}

In recent years, global climate change has become increasingly significant, and natural disasters have occurred frequently, hindering social and national development. We are aware that climate change has a huge impact on people's lives. It will cause social costs to be damaged and government institutions to be unstable, which will lead to the country's fragility. The top 10 most vulnerable countries (South Sudan, Somalia, the Central African Republic, Yemen, the Sudan, Syria, the Democratic Republic of the Congo, Chad, Afghanistan, and Iraq) cited as an example how to find out how climate change has increased the vulnerability of a country.

\section{The Country's Vulnerability Index}

This paper selects the vulnerability index and its corresponding average temperature in 10 countries (South Sudan, Somalia, Central African Republic, Yemen, Sudan, Syria, Democratic Republic of the Congo, Chad, Afghanistan, and Iraq), taking the first South Sudanese country as an example., as shown in Table 1.

Table 1 Vulnerability index

\begin{tabular}{|c|c|c|c|c|c|c|c|c|c|c|c|c|c|c|}
\hline Year & Rank & Total & C1: SA & C2: FE & C3: GG & E1: Ec & E2: EI & E3: HF\&BD & P1: SL & P2: PS & P3: HR & S1: DP & S2: R\&IDP & X1: EI \\
\hline 40909 & n/r & 108.4 & 9.7 & 10 & 9.9 & 7.3 & 8.8 & 6.4 & 9.1 & 9.5 & 9.2 & 8.4 & 9.9 & 10 \\
\hline 41275 & 4 th & 110.6 & 9.6 & 9.8 & 10 & 8.6 & 8.9 & 6.5 & 9.1 & 9.8 & 9.3 & 8.9 & 10 & 10 \\
\hline 41640 & 1st & 112.9 & 9.9 & 10 & 10 & 8.8 & 8.9 & 6.8 & 9.7 & 9.9 & 9.9 & 9.1 & 10 & 9.9 \\
\hline 42005 & 1st & 114.5 & 10 & 10 & 10 & 9 & 8.8 & 6.9 & 10 & 10 & 10 & 9.8 & 10 & 10 \\
\hline 42370 & 2nd & 113.8 & 10 & 9.7 & 9.9 & 9.3 & 9 & 6.6 & 9.7 & 10 & 9.7 & 9.9 & 10 & 10 \\
\hline 42736 & 1st & 113.9 & 10 & 9.7 & 9.7 & 10 & 8.9 & 6.4 & 10 & 10 & 9.5 & 9.9 & 10 & 9.8 \\
\hline
\end{tabular}


This paper uses the four major indicators of security, economy, politics, and society to measure the vulnerability of a country. The four indicators are further divided into these 12 small indicators: security institutions, upper echelons, group dissatisfaction, economic, economic inequality, population migration and brain drain, national (political) legitimacy, public services, people's rights, total population pressure the number of exiles and external interventions are very statistically significant.

Using the correlation coefficient model to study the rise in the average temperature of the unit is vulnerable to the country. The model is as follows.

$$
\Delta E=\sum_{i=1}^{n} k_{i}
$$

Where ki is the correlation coefficient between the average temperature and 12 indicators.

Use MATLAB to produce the following correlation coefficient diagram for the average temperature and 12 indicators, as shown in Figure 1.

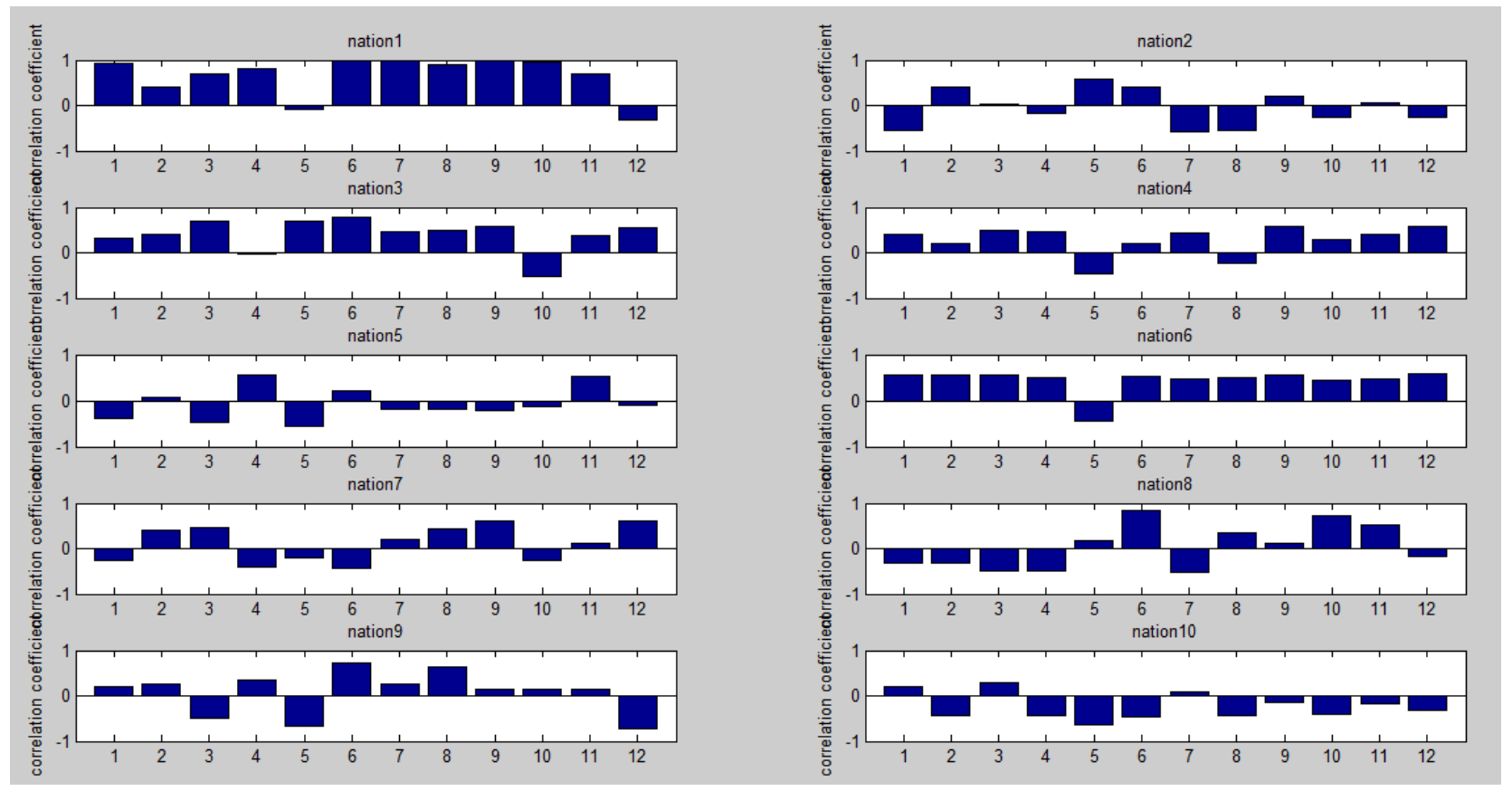

Figure 1 Correlation coefficient between average temperature and 12 indicators

\section{The Relevance of Climate Change and National Vulnerability}

Because the weights are consistent with the weights of the comprehensive score indicators of the 12 national vulnerable indicators, the ranking and comparison indicators are directly accumulated. The greater the index after the summation, the higher the global temperature is, which brings about an unfavorable trend to the stability of the country and is more likely to cause the formation of the country's vulnerability. A positive evaluation indicator indicates that the climate change has harmful effects. On the contrary, it has good effect. The comprehensive evaluation index of national vulnerability caused by climate change is shown in Figure 2.

From the graph analysis, after taking the logarithm, a negative value was taken. Therefore, the larger the index value, the higher the global temperature will bring favorable trend to the country's stability. According to the climate change given by the model, the impacts of these ten countries' vulnerability are ranked. The analysis shows that: $E$ is a positive value, which means that it brings about harmful effects, which leads to an increase in the comprehensive index of national vulnerability; On the contrary, it has a beneficial effect on the stability of the country. 


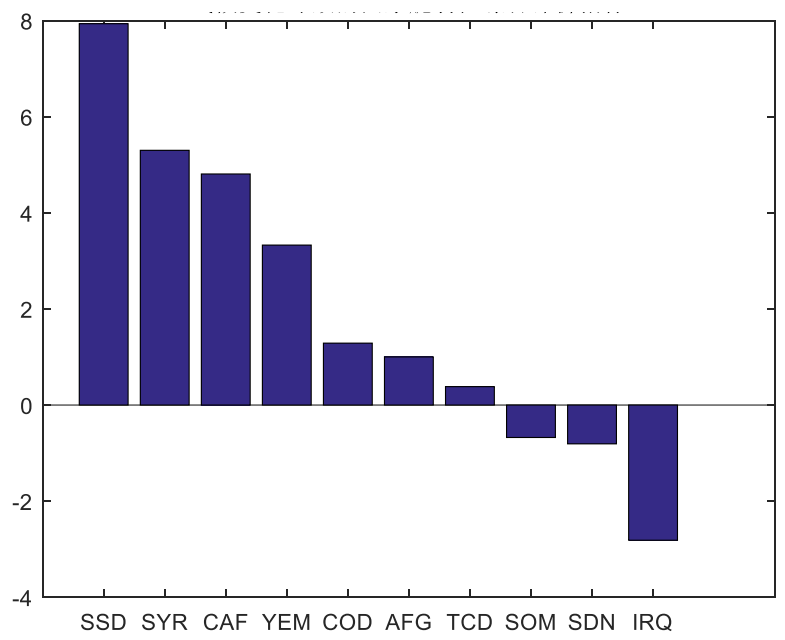

Figure 2. Comprehensive Assessment Index of National Vulnerability Caused by Climate Change

\section{Comprehensive Evaluation Model of State Vulnerability}

Considering the current climate-specific changes (global warming) under the multi-index (12 indicators) weight modification model, the revised national vulnerability assessment has become a percentage system scoring mechanism, 60 is divided into national vulnerability delineation standards, 40 is classified as very fragile.

The original national vulnerability comprehensive assessment model is an equal weight model that takes into account 12 factors. This model does not consider the impact of global warming and climate change.

$$
E_{\mathrm{j}}=\sum_{j=1}^{m} y_{i j}
$$

Among them, $\mathrm{j}$ is the comprehensive score of the $\mathrm{jth}$ country; $\mathrm{ij}$ is the ith factor of the $\mathrm{jth}$ country.

Taking the impact of climate change into account, global warming will affect the changes in 12 indicators. In order to quantify this change, this effect was calculated for each of the different countries and the changes in weights of the 12 factors caused by climate warming were separately calculated.

It is assumed that the weight is 1 when climate change is not considered, and the weights after considering climate change $\omega_{\mathrm{ij}}$ changes to:

$$
\omega_{\mathrm{ij}}=\frac{1+k_{i j}}{\sum_{i=1}^{12}\left(1+k_{i j}\right)}
$$

$k_{\mathrm{ij}}$ denotes the correlation coefficient between climate warming and factor $\mathrm{i}$ in the $\mathrm{jth}$ country $k_{\mathrm{ij}}$

takes $(-1,1)$, divided into positive and negative correlations. Positive correlation indicates that warming will exacerbate the impact of this factor on the country's fragility. On the contrary, it weakens.

\section{Conclusion}

This model standardizes data in different dimensions, and meanwhile it uses the established rules and correlation coefficients to determine the correlation and impact of weather changes and country fragility. These analyses can suggest which climatic factors the country should focus on and when to take the necessary interventions. At the same time, after ranking the vulnerability index and the correlation coefficient, the countries that are at the top of the list will also receive attention. 


\section{References}

[1]. Information on: Late Cenozoic Climate Change in Monsoon-Arid Asia and Global Changes.

[2]. Information on: CLIMATE CHANGE IMPACT ON CLIFF INSTABILITY AND EROSION.

[3]. Information on: An approach to assess flooding and erosion risk for open beaches in a changing climate.

[4]. Information on: Global food security under climate change.

[5]. Information on: Fragile State Index Trends and Developments. A Partial Order Data Analysis. 